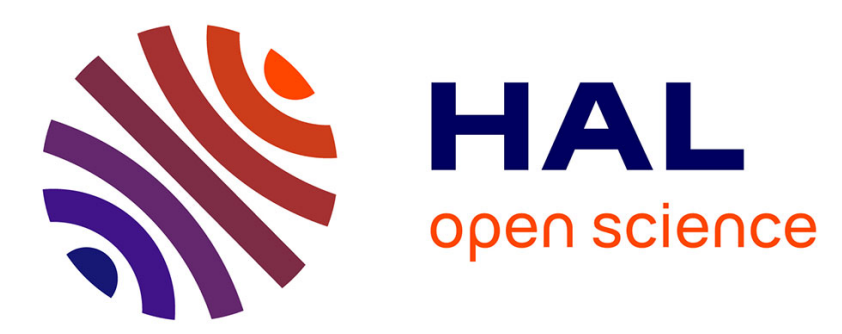

\title{
IRIS FEATURES EXTRACTION USING WAVELET PACKETS
}

Erik Rydgren, Thomas Ea, Frédéric Amiel, Florence Rossant, Amara Amara

\section{To cite this version:}

Erik Rydgren, Thomas Ea, Frédéric Amiel, Florence Rossant, Amara Amara. IRIS FEATURES EXTRACTION USING WAVELET PACKETS. ICIP, Oct 2004, Singapore, Singapore. 10.1109/ICIP.2004.1419435 . hal-02882950

\section{HAL Id: hal-02882950 https://hal.science/hal-02882950}

Submitted on 28 Jun 2020

HAL is a multi-disciplinary open access archive for the deposit and dissemination of scientific research documents, whether they are published or not. The documents may come from teaching and research institutions in France or abroad, or from public or private research centers.
L'archive ouverte pluridisciplinaire HAL, est destinée au dépôt et à la diffusion de documents scientifiques de niveau recherche, publiés ou non, émanant des établissements d'enseignement et de recherche français ou étrangers, des laboratoires publics ou privés. 


\title{
IRIS FEATURES EXTRACTION USING WAVELET PACKETS
}

\author{
Erik RYDGREN, Thomas EA, Frédéric AMIEL, Florence ROSSANT, Amara AMARA \\ Institut Supérieur d'Electronique de Paris, ISEP \\ 21 rue d'Assas \\ 75270 Paris Cedex 06 \\ erik.rydgren | thomas.ea | frederic.amiel | florence.rossant | amara.amara@isep.fr
}

\begin{abstract}
This article presents an application of wavelet packet analysis to the features extraction part of an iris recognition system. An energy measure is used to identify the particular packets that carries discriminating information about the iris texture. Several different orthogonal wavelets are tested and a comparison to nonorthogonal analysis using Gabor wavelets is done. The experimental results show $100 \%$ correct classifications when applying the algorithm on an iris image database and the new algorithm is therefore an interesting alternative to Gabor based methods.
\end{abstract}

\section{INTRODUCTION}

Biometric measures such as iris and retina texture, palmprint texture, face geometry and fingerprints is an interesting alternative to traditional authorization techniques such as keys and codes. Good biometric identification systems are well protected against forgery and hacking while also offering high user convenience.

Iris recognition technology has been a field of intensive research the last 15 years. The first system was patented by Daugman in 1994 [2]. Daugman's system is based on non-orthogonal wavelet analysis using so called Gabor wavelets [3], and has emerged as something of a benchmark in the field. Since then, much further work has been done by several research groups such as Zhu et al. [10], Tisse et al. [9] and Wildes [11]. Lim et al. has proposed a system based on orthogonal wavelet transform using the Haar wavelet [6]. Lim et al. uses the diagonal detail $(\mathrm{HH})$ coefficients as iris signature. However, it will be shown in this paper that the wavelet packet approach can be a good alternative to the standard wavelet transform since it offers a more detailed division of the frequency plane.

The outline of this paper is as follows. The preprocessing part of the system is presented in section 2 . In this case preprocessing refers to every operation that takes place before the features extraction. Section 3 introduces the two different extraction techniques used: wavelet packets versus Gabor wavelets. Section 4 presents the experimental results acquired with each of the two methods. Finally, some conclusions and proposed future work can be found in section 5 .

\section{PREPROCESSING}

The images used for this study are acquired by Miles Research [7] using dedicated iris imaging equipment providing very little eyelid and eyelash occlusion and also little light reflections. The images contain some of the surroundings of the iris which must be removed before further processing. This is done by a localization process where the center coordinates and radius for the pupil and iris are found. The iris texture is then transformed into a polar representation. The last step of the preprocessing procedure is to increase the contrast of the iris texture by histogram equalization. Figure 1 summarises the different preprocessing steps.

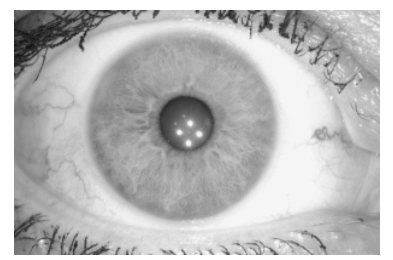

(a)

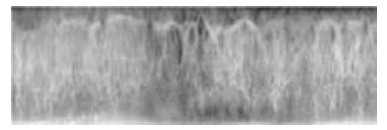

(c)

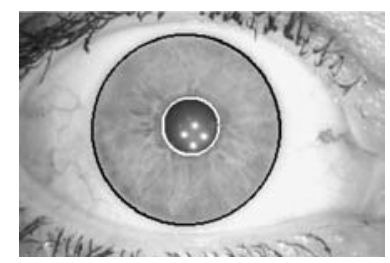

(b)

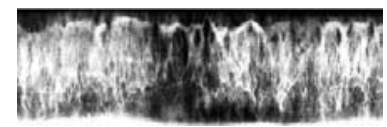

(d)
Figure 1. a) An eye photo before processing, b) a located iris, c) iris texture after unwrapping, d) unwrapped iris texture after histogram equalization

\subsection{Image localization and equalization}

The iris localization algorithm uses the fact that there is a relatively high contrast between the iris and the surrounding white sclera tissue. A simple gradient based search method has therefore proven successful. The pupil is located either by a similar gradient based method, or by isolating the gray-level values on the pupil through evaluating the histogram. 


\subsection{Unwrapping}

The geometry of the iris is circular and most of its interesting textural features are extended in the radial and, to a lower extent, the angular direction. Therefore analysis is simplified by an adapted polar transform, suggested by Daugman [3]. The adaptation is motivated by the fact that the pupil and iris is not necessarily concentric, the pupil is often somewhat displaced towards the nose and downwards, and also since the pupil diameter is not constant. This is amended by a transform that normalizes the distance between the pupil boundary and the outer iris boundary. Such a transform is expressed by equation 1 , where $\left(\mathrm{x}_{\mathrm{p}}, \mathrm{y}_{\mathrm{p}}\right)$ and $\left(\mathrm{x}_{\mathrm{i}}, \mathrm{y}_{\mathrm{i}}\right)$ are a pair of coordinates on the pupil and iris border. The figure to the left of equation 1 defines the angle variable $\theta$ and the radius variable $r$. The radius is normalized to the interval $[0,1]$.

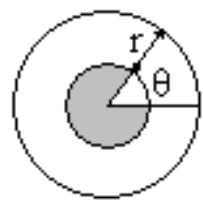

$$
\begin{aligned}
& x(r, \theta)=r x_{i}(\theta)+(1-r) x_{p}(\theta) \\
& y(r, \theta)=r y_{i}(\theta)+(1-r) y_{p}(\theta)
\end{aligned}
$$

The unwrapped image is then histogram equalized to increase the contrast of the texture [5].

\section{FEATURES EXTRACTION}

\subsection{Wavelet packets}

Wavelet packet decompositions are usually calculated using orthonormal or biorthogonal wavelets. In that case the decomposition can be implemented using the fast wavelet transform algorithm. At each stage in the decomposition part of a two-dimensional FWT filter bank, four output images are generated. The images contain approximation $(\mathrm{A})$, horizontal detail $(\mathrm{H})$, vertical detail (V) and diagonal detail (D) coefficients respectively. In wavelet packet analysis, each of these output images are further processed as opposed to wavelet analysis where only the approximation image is further processed. The result is that the whole frequency plane is splitted into equally sized bands [1].

A 3-level wavelet packet decomposition generates 64 output subimages, each representing a part of the frequency plane. Figures 2 and 3 illustrates the wavelet packet decomposition structure and the frequency plane respectively.

An approximative energy distribution for an image can be calculated using wavelet packets and the energy measure:

$$
E_{i}=\sum_{j, k} w_{i}(j, k)^{2}
$$

where $E_{i}$ is the energy of the subimage $w_{i}$. Experiments shows that the energy measure (2), applied to the wavelet packets, gives a good description of the frequency content of an image.

The subimages in the wavelet packet tree holds information about both frequency (scale) and localization as opposed to for example the Fourier transform that only hold frequency information. The subimages are therefore suitable candidates for iris signatures. A compact signature can be achieved by quantizing the wavelet coefficients into one bit each, depending on their sign.

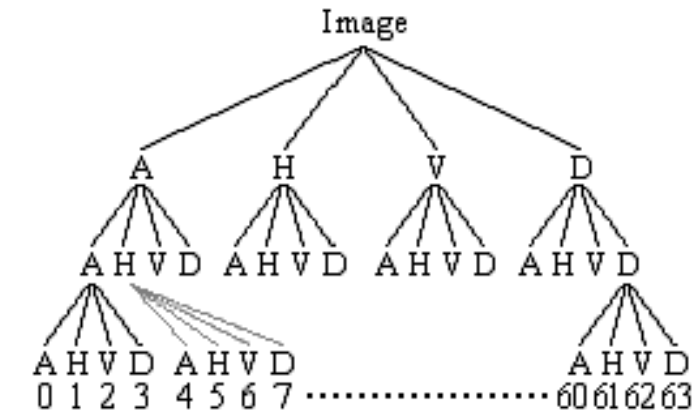

Figure 2. A wavelet packet tree. Only three of the 16 possible third-level decompositions are displayed.

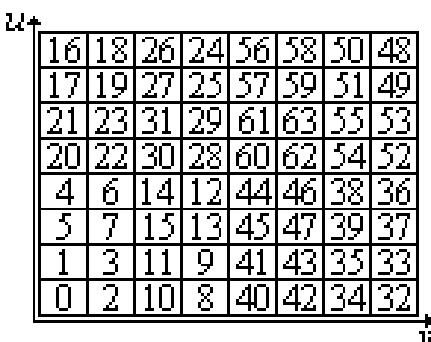

Figure 3. The frequency plane $(u, v)$ division corresponding to a complete three-level wavelet packets transform.

\subsection{Gabor phase}

Gabor wavelets are defined as gaussians modulated by complex exponentials. It has been shown that Gabor wavelets offer maximum conjoint space-frequency resolution [4]. Further, Gabor wavelets have the same functional form in spatial and frequency domain.

The Gabor wavelets used in image processing are two-dimensional, with a certain frequency and direction of the complex exponential. However, when the complex exponential propagates in a direction perpendicular to or diagonal to the coordinate axes, it is possible to express the two-dimensional wavelet as the outer product of two one-dimensional wavelets. This makes it possible to implement the Gabor decomposition in an efficient way using 1D filters [8].

The phase of the complex-valued decomposition is used as iris signature. Both the real and imaginary parts of the decomposition coefficients are evenly distributed 
around 0 , and a coarse local phase measure is therefore given by the sign of the coefficients [3].

\section{EXPERIMENTAL RESULTS}

Table 1 to 3 presents some results using the two different features extraction techniques. The tests was conducted in the following way. A signature was extracted from each of the 82 photos in the database. The photos was taken from 33 different eyes and thus there were more than one photo per each eye. The size of the unwrapped textures was set to $128 * 512$ pixels when using the wavelet packet method and $64 * 256$ when using the Gabor method. The reason for using a larger texture for the wavelet packet method is that the subimages at level three are downsampled by a factor $2^{3}=8$ and as a result, the signature size would otherwise be too small. The signature size for the two methods were 896 bits (wavelet packets) and 2684 bits (Gabor). Each signature was compared to all the other signatures using the Hamming distance, given by:

$$
H D=\frac{1}{N} \sum_{j, k} S_{1}(j, k) \oplus S_{2}(j, k)
$$

where $S_{1}$ and $S_{2}$ are two binary signatures, $N$ is the total number of bits in each signature and $\oplus$ is the XOR operator defined as:

$$
x \oplus y= \begin{cases}0 & x=y \\ 1 & x \neq y\end{cases}
$$

where $x$ and $y$ are single bits. The Hamming distance is thus a measure for the similarity between two bit sequences, the lower Hamming distance the higher similarity.

It is very important that the system is rotational invariant since any tilt of the head when taking the iris photos will produce a rotation in the iris texture. Two different methods to produce rotational invariance was tried. First, the actual photos was shifted to produce several signatures for every photo. Secondly, the signatures themselves were shifted. The two methods proved to yield equivalent results, but the second one is more effecient and therefore preferred.

For the wavelet packet features extraction, subimage number 2 (see figure 2 and 3 ) was used as signature. This was motivated by an energy measurement using eq. (2) that showed that most of the texture information is encoded by this subimage, see figure 4 for a plot giving the mean energy distribution for 25 unwrapped iris images from different eyes. The energy corresponding to subimage 0 has been removed since this subimage contains offset (DC) information. From the frequency plane mapping of figure 3 it is clear that subimage 2 codes relatively low frequency energy close to the horizontal axis. The unwrapped iris images are characterised by lines extending in the vertical direction, and therefore contain much energy at horizontal frequency. Figure 4 shows that subimage 10 holds much energy as well. It is therefore interesting to also evaluate the possibilities to use this subimage as a signature.

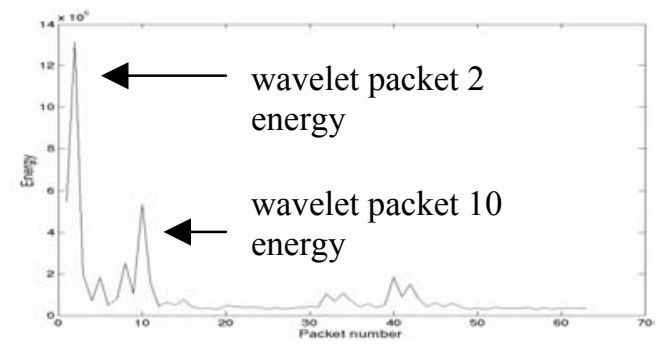

Figure 4. Mean wavelet packet energy distribution for 25 unwrapped iris textures. The indexes on the $x$-axis corresponds to the frequency plane in figure 3.

The discrimination results using wavelet packets are found in table 1 and 2. The first column (Wavelet) indicates which wavelet was used and columns 2 to $5\left(\mu_{\mathrm{a}}\right.$, $\left.\sigma_{\mathrm{a}}, \mu_{\mathrm{i}}, \sigma_{\mathrm{i}}\right)$ presents first and second order statistics for the distribution of Hamming distances for authentics and imposters respectively. The authentic distances are from different signatures from the same individual while the imposter distances are from different individuals. The last column (Sep.) gives the separation between the two distributions, that is the distance between the largest authentic measurement and the smallest imposter measurement. The separation should be a positive number, the larger the better.

\begin{tabular}{|c|c|c|c|c|c|}
\cline { 2 - 5 } \multicolumn{1}{c|}{} & \multicolumn{2}{c|}{ Authentics } & \multicolumn{2}{c|}{ Imposters } & \multicolumn{1}{c|}{} \\
\hline Wavelet & $\boldsymbol{\mu}_{\mathbf{a}}$ & $\boldsymbol{\sigma}_{\mathbf{a}}$ & $\boldsymbol{\mu}_{\mathbf{i}}$ & $\boldsymbol{\sigma}_{\mathbf{i}}$ & Sep. \\
\hline Haar & 0.185 & 0.051 & 0.457 & 0.011 & 0.071 \\
\hline Daubechies2 & 0.202 & 0.058 & 0.459 & 0.010 & 0.040 \\
\hline Daubechies4 & 0.208 & 0.056 & 0.460 & 0.010 & 0.060 \\
\hline Daubechies6 & 0.220 & 0.054 & 0.461 & 0.010 & 0.046 \\
\hline Daubechies8 & 0.224 & 0.058 & 0.461 & 0.010 & 0.018 \\
\hline biorthog. 1.3 & 0.186 & 0.053 & 0.459 & 0.010 & 0.076 \\
\hline biorthog. 2.6 & 0.240 & 0.066 & 0.463 & 0.008 & 0.000 \\
\hline biorthog. 3.9 & 0.270 & 0.067 & 0.463 & 0.009 & -0.017 \\
\hline coiflet 2 & 0.214 & 0.061 & 0.460 & 0.010 & 0.026 \\
\hline coiflet 4 & 0.216 & 0.055 & 0.462 & 0.010 & 0.060 \\
\hline symlet 2 & 0.202 & 0.058 & 0.460 & 0.010 & 0.040 \\
\hline symlet 4 & 0.213 & 0.061 & 0.460 & 0.011 & 0.027 \\
\hline symlet 6 & 0.215 & 0.062 & 0.460 & 0.010 & 0.019 \\
\hline
\end{tabular}

Table 1. Results using wavelet packets with various wavelets. Notation: $\boldsymbol{\mu}$ - mean, $\boldsymbol{\sigma}$ - std. dev.

From table 1 it is clear that the Haar and Biorthogonal 1.3 wavelets are most suitable in this application. The imposters mean value is slightly below 0.5 . This is due to 
the rotation invariance implementation, where the signatures are shifted relative to eachother and the best match (lowest Hamming) for all shifts is recorded.

The discrimination performance of subimage 10 was also tested, the results can be found in table 2 .

\begin{tabular}{|c|c|c|c|c|c|}
\cline { 2 - 5 } \multicolumn{1}{c|}{} & \multicolumn{2}{c|}{ Authentics } & \multicolumn{2}{c|}{ Imposters } & \multicolumn{1}{c}{} \\
\hline Wav. & $\boldsymbol{\mu}_{\mathbf{a}}$ & $\boldsymbol{\sigma}_{\mathbf{a}}$ & $\boldsymbol{\mu}_{\mathbf{i}}$ & $\boldsymbol{\sigma}_{\mathbf{i}}$ & Sep. \\
\hline Haar & 0.276 & 0.059 & 0.461 & 0.009 & 0.000 \\
\hline bior1.3 & 0.274 & 0.060 & 0.462 & 0.008 & 0.010 \\
\hline
\end{tabular}

Table 2. Results using subimage 10.

Although the separation between the authentics and imposters distributions is smaller when using subimage 10 than subimage 2 , the means are clearly separated and there is thus discriminating information also in this subimage.

Table 3 gives the results from experiments with Gabor wavelets. The optimal frequency for the modulating complex exponential was found to be $f$ $=0.021 f_{s}$, where $f_{s}$ is the sampling frequency in rad/pixel.

\begin{tabular}{|c|c|c|c|c|c|}
\cline { 2 - 5 } \multicolumn{1}{c|}{} & \multicolumn{2}{c|}{ Authentics } & \multicolumn{2}{c|}{ Imposters } & \multicolumn{1}{c}{} \\
\hline $\boldsymbol{f}$ & $\boldsymbol{\mu}_{\mathrm{a}}$ & $\boldsymbol{\sigma}_{\mathrm{a}}$ & $\boldsymbol{\mu}_{\mathbf{i}}$ & $\boldsymbol{\sigma}_{\mathbf{i}}$ & Separ. \\
\hline 0.021 & 0.106 & 0.032 & 0.445 & 0.018 & 0.207 \\
\hline
\end{tabular}

Table 3. Results using Gabor wavelets of frequency $f$.

Figure 5 show the histograms for authentics and imposters Hamming distance distributions, using wavelet packets and Gabor wavelets. The upper and lower left histograms shows the authentic and imposter distributions for the wavelet packet algorithm using subimage 2 as signature and the biorthogonal 1.3 wavelet. The upper and lower right histograms shows the corresponding distributions in the Gabor case.

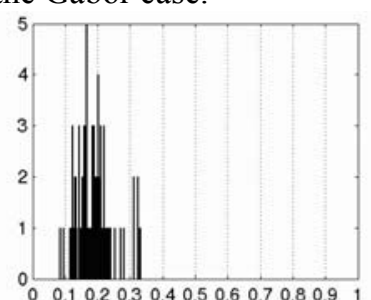

(a)

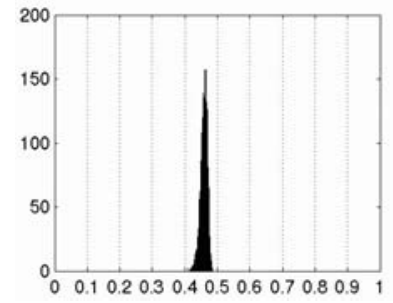

(b)

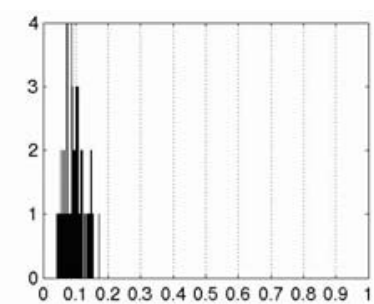

(c)

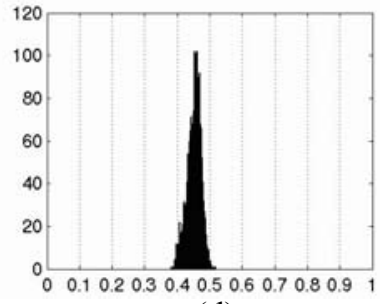

(d)
Figure 5. Hamming distance distributions for authentics and imposters using wavelet packets (a), (b) and Gabor wavelets (c),(d)

\section{CONCLUSIONS AND FUTURE WORK}

A wavelet packet approach to iris recognition has been proposed and evaluated. The algorithm manages to correctly identify all authentic irises and dismiss all imposters. Since the algorithm is based on orthogonal analysis, the fast wavelet transform can be used to achieve high processing efficiency. The calculation complexity is particularily low if the Haar wavelet is used because of the simple structure of the associatied filter. The performance for the benchmark Gabor wavelet is so far superior but it is likely that the performance of the wavelet packets algorithm can be increased in the future. The signatures extracted with wavelet packet analysis is also more compact than the signatures extracted using the Gabor method. Some propositions for future work includes evaluating the possibilities of using more than one subimage and a longer signature. The performance might also be increased by using another distance measure than the Hamming distance, such as the weighted Euclidian distance, or by using more than 3 levels of transformation.

\section{ACKNOWLEDGEMENTS}

The authors are grateful to Jon Miles at Miles Research [7] for providing the iris image database used in this study.

\section{REFERENCES}

[1] J. Bergh, F. Ekstedt and M. Lindberg, Wavelets, Studentlitteratur, Lund, 1999

[2] "Biometric Personal Identification System Based on Iris Analysis.", U.S. Patent No. 5,291,560 issued March 1, 1994 (J. Daugman)

[3] J. Daugman, "High confidence visual recognition of persons by a test of statistical independence.", IEEE Trans. PAMI, vol. PAMI-15, pp. 1148-1161, 1993

[4] J. Daugman, "Uncertainty relation for resolution in space, spatial frequency and orientation optimized by two-dimensional visual cortical filters." Opt. Soc. Amer. A, Vol 2, pp. 1160-1169, 1985

[5] R. C. Gonzales and R. E. Woods, Digital Image Processing, Prentice Hall, New Jersey, 2002

[6] S. Lim et al., "Efficient iris recognition through improvement of feature vector and classifier", ETRI J., Vol 23, pp. 61-70, 2001

[7] Miles Research, www.milesresearch.com

[8] O. Nestares et al., "Efficient spatial-domain implementation of a multiscale image representation based on Gabor functions", J. of Electronic Imaging, Vol 7, pp. 166-173, 1998

[9] Tisse et al., "Person Identification technique using human iris recognition", Proc.15th Int. Conf. on Vision Interface, Calgary, Canada, 2002

[10] Y. Zhu, T. Tan and Y. Wang, "Biometric personal identification based on iris patterns", Proc. 15th Int. Conf. on Pattern Recognition, Barcelona, Spain, 2000

[11] R. P. Wildes, "Iris recognition: an emerging biometric technology", Proc. IEEE 85, Vol 9, pp. 1348-1363, 1997 